\title{
Astrometric Galactic maser measurements cross-matched with Gaia
}

\author{
L.H. Quiroga-Nuñez ${ }^{1,2}$ H.J. van Langevelde ${ }^{2,1}$ M.J. Reid ${ }^{3}$ \\ L.O. Sjouwerman ${ }^{4}$ Y.M. Pihlström ${ }^{5}$ A.G.A. Brown ${ }^{1}$ and J.A. Green ${ }^{6}$ \\ ${ }^{1}$ Leiden Observatory, Leiden University, \\ P.O. Box 9513, 2300 RA Leiden, The Netherlands. \\ email: quiroganunez@strw.leidenuniv.nl \\ ${ }^{2}$ Joint Institute for VLBI ERIC (JIVE), \\ Postbus 2, 7990 AA Dwingeloo, The Netherlands. \\ ${ }^{3}$ Harvard-Smithsonian Center for Astrophysics, \\ 60 Garden Street, Cambridge, MA 02138, USA. \\ ${ }^{4}$ National Radio Astronomy Observatory, \\ P.O. Box 0, Lopezville Road 1001, Socorro, NM 87801, USA. \\ ${ }^{5}$ Department of Physics and Astronomy, University of New Mexico, \\ MSC07 4220, Albuquerque, NM 87131, USA. \\ ${ }^{6}$ CSIRO Astronomy and Space Science, Australia Telescope National Facility, \\ PO Box 76, Epping, NSW 1710, Australia.
}

\begin{abstract}
Using the VLBA, the BeSSeL survey has provided distances and proper motions of young massive stars, allowing an accurate measure of the Galactic spiral structure. By the same technique, we are planning to map the inner Galaxy using positions and velocities of evolved stars (provided by the BAaDE survey). These radio astrometric measurements (BeSSeL and BAaDE) will be complementary to Gaia results and the overlap will provide important clues on the intrinsic properties and population distribution of the stars in the bulge.
\end{abstract}

Keywords. Galaxy: bulge, stars: AGB and post-AGB, masers, astrometry.

\section{Context and aim}

By monitoring the position of a maser bearing star in the sky with respect to a background extragalactic source, it is possible to estimate its proper motion and parallax (e.g., Honma et al., 2008). Maser astrometric measurements of high-mass star forming regions using VLBI have recently made substantial progress on our understanding of the Milky Way (Reid \& Honma, 2014, Reid et al., 2014). The Bar and Spiral Structure Legacy (BeSSeL) survey has obtained accurate positions, proper motions, line-of-sight velocities and distances to more than 100 young massive stars, which are associated with water and methanol maser emission. Using this $6 \mathrm{D}$ phase space information, the Galactocentric distance, solar rotation speed, rotation curve and solar motion have been refined (Reid et al., 2014). Moreover, simulations of the Galactic distribution of masers confirmed the accuracy of the parameter values found by BeSSeL (Quiroga-Nuñez et al., 2017a).

The Bulge Asymmetries and Dynamical Evolution (BAaDE) project surveys evolved stars with $\mathrm{SiO}$ maser emission at 43 and $86 \mathrm{GHz}$ using the VLA and ALMA. BAaDE will eventually provide accurate positions and radial velocities for 20,000 stars mainly in the bulge (Sjouwerman et al., 2016). In principle, it is possible to estimate proper motions and parallaxes for some BAaDE targets following the technique used in BeSSeL. Moreover, since these radio astrometric measurements are made in the Galactic plane but are not affected by extinction, they can provide complementary information where optical surveys cannot reach. This will help to characterize the stellar population of the inner Galaxy, which is crucial to understand the Galactic dynamical evolution. 

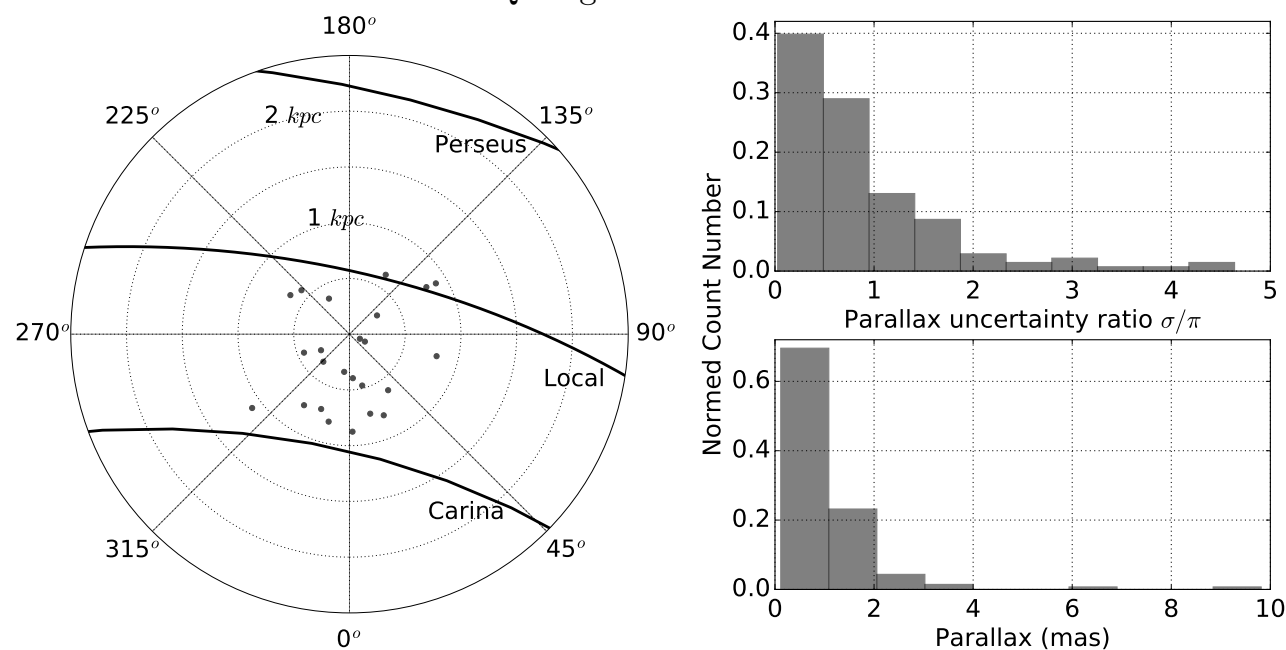

Figure 1. Left: Distribution of the sample obtained by cross-matching BAaDE targets and TGAS over a top view of the Milky Way centered in the sun's position. Only the sources with $\sigma / \pi \leqslant 0.3$ are plotted. Right: Distribution of the parallax uncertainty ratio and the parallax for the cross-match BAaDE-TGAS sample. Only the sources with $\sigma / \pi \leqslant 5$ are plotted.

\section{Cross-matching with Gaia (TGAS) and future work}

Quiroga-Nuñez et al., 2017b have cross-matched BAaDE targets with Gaia DR1 resulting in more than 2,000 matches, where false positives were avoided by using several filters (distance analysis, IR color filters and variability). From that sample, 156 sources were observed with Hipparcos and therefore are part of TGAS. The Galactic distribution of these sources is shown in the left panel of Fig. 1, where only sources with low parallax uncertainties $(\sigma / \pi<0.3)$ are shown. They represent $15 \%$ of the sample, and for those, we used the distance-parallax definition without prior assumption (Bailer-Jones, 2015). Right panel of Fig. 1 shows the distribution of the parallax uncertainty ratio $(\sigma / \pi)$ and the parallax for those sources with $\sigma / \pi<5$. They represent $88 \%$ of the sample. As it can seen in Fig. 1 the sample is confined to the foreground Galaxy.

We will soon have an unique sample of $\sim 2,000$ evolved stars with radio (positions, radial velocities, $\mathrm{SiO}$ maser emission lines and rates from $\mathrm{BAaDE}$ ), infrared ( $J, H, K$ filters from 2MASS) and optical (parallaxes, proper motions, periods and photometry from Gaia) bands to characterize the stellar population in the Galactic bulge. In addition, VLBI campaigns are being defined to provide astrometric information of AGB stars with bright masers and hence, some individual stars can be studied in detail. Finally, a fundamental comparison between radio and optical parallax-distance estimate can be performed.

\section{Acknowledgements}

This work has used data from the ESA mission Gaia, processed by the Gaia DPAC. This material is based upon work supported by the NSF under grant 1517970 .

\section{References}

Bailer-Jones, C. A. L. 2015, PASJ, 127, 994

Honma, M., Tamura, Y. \& Reid, M. J. 2008, PASJ, 60, 951

Quiroga-Nuñez, L. H., van Langevelde, H. J., Reid, M. J. \& Green, J. A. 2017a, A\&A, 604, A72

Quiroga-Nuñez, L. H., van Langevelde, et al. 2017b, Proc. IAU Symposium, 330 in press

Reid, M. J. \& Honma, M 2014, ARA\&A, 52, 339

Reid, M. J., Menten, K. M., Brunthaler, Zheng, X. W., et al. 2014, ApJ, 783, 130

Sjouwerman, L. O., Pihlström, Y. M., Rich, R. M., et al. 2016, Proc. IAU Symposium, 322, 103 